le nombre de plots de freinage sera en général déterminé à l'avance par la disposition du combinateur, par lc nombre de plots de démarrage (le nombre total de plots ne devant pas dépasser une certaine limite), etc.

En second lieu, par suite de la diversité des arrêts, il est difficile d'assigner aux intensités limites des valeurs bien déterminécs. Quant aux valeurs des résistances correspondaut à chaque cran du combinateur, l'on pourra les détermiuer, soit expérimentalement, soit d'après l'épure de la fig. 9 , en se donnant les vitesses réalisables sur chaque pente du parcours. Ici encore la solution pourra être donnée d'avance,surtout si l'ou se sert pour le freinage des résistances de démarrage, et il n'est guère possible de fixer des règles géuérales.

Considérations pratiques. - Actuellement, l'on se sert en général, pour la manœuvre des freins électriques, des cylindres munis déjà de touches pour le réglage de la vitesse.

Les plots destinés au freinage sont absolument identiques aux autres; aussi n'entrerons-nous pas dans les détails de construction des combinateurs, renvoyant nos lecteurs que la question intéresse aux traités spéciaux (*). Nous attirerons cependant l'attention sur un point qui peut avoir son intérêt en pratique: les combinateurs actuels comportent, en général, deux manettes (fig. I 2), l'une $M$ servant à la mise en marche et au réglage de la vitesse (par le couplage série parallèle par exemple), l'autre plus petite $\mathrm{N}$ servant à obtenir les marches avant etarrière, suivant qu'elle occupe les positions A et B. Par laquelle doit-on commander le frein électrique? Si l'on se sert de la manette M, l'on obtient une rapidité et une facilité de manœuvre très grandes, puisque le mécanicien n'a, pour frei-

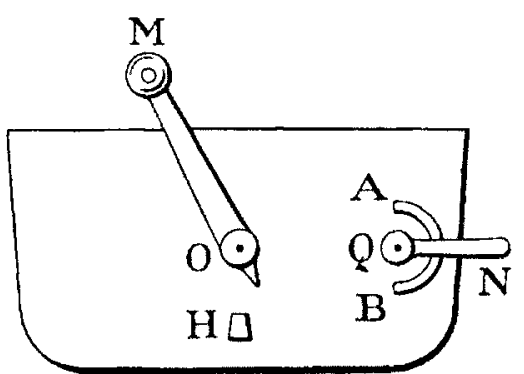

Fig. I 2 ner, qu'à continuer le mouvement de la manette $\mathrm{M}$, après l'avoir ramenée au zéro.

Par contre, le freinage par les moteurs exigeant le renversement des connexions entre induits et inducteurs, comme pour la marche arrière (**), l'on est amené ainsi à mettre des plots d'inversion sur le cylindre de couplage commandé par la manette $\mathrm{M}$, ce qui complique le combinatcur. Enfin, celui-ci devant fournir tous les crans de marche et de freinage pour un tour de la manette $M$ au maximum, l'ón conçoit que pour passer d'un plot à l'autre ledéplacement angulaire sera moins grand, ce qui facilite la production d'arcs. Au contraire, si j'on se sert de la manette $\mathrm{N}$, les connexions sont notablement simplifiées, le cylindre de couplage correspondant produisant, pour la marche arrière, l'inversion de couplage nécessaire au freinage. Il semble done, qu'en résumé, l'emploi de la

(1) Voir notamment le magistral traité déjà cité de MM. A, Blondel et $P$. Dubois, ainsi que l'ouvrage plus récent de MM. Barbillion tt Griffisch: "Traité pratique de traction électrique $»$.

(*) Ceci a lieu pour le cas le plus fréquent des moteurs-série. manette $\mathrm{M}$ est préférable sur les tramways ou les arrêts sont fréquents et exigent une grande facilité de manœuvre; sur les voitures de chemins de fer électriques, au contraire, l'on emploiera la manette $\mathrm{N}$ qui permet plus facilement l'usage d'une tension élevée (80o à tooo volts).

Conclusions. - Comme on vient de le voir par tout ce qui précède, le freinage par les moteurs marchant en génératrices sur des résistances, a les grands avantages de la simplicité et de la parfaite modérabilité sur les pentes. On lui a reproché les deux inconvénients suivants:

$I^{\circ}$ Il fait travailler fortement les engrenages dans des conditions défavorables, le pignon du moteur étant alors commandé par la roue dentée de l'essieu. Il convient cependant de remarquer que lorsque les moteurs ne sont pas freinés, les dents ont aussi, par suite de l'inertie des induits, un effort considérable à subir lors d'un arrêt rapide.

$2^{0}$ Il est parfois d'un emploi peu commode pour les arrêts complets, lorsque les moteurs mettent de la paresse à s'amorcer. Le mécanicien, sentant que l'amorcement ne se fait pas au premier cran, passe aux suivants ; l'amorcement peut se faire alors brusquement, à la grande incommodité des voyageurs. Pour remédier à cet inconvénient, il suffit en général de tenir en bon état les collecteurs, un mauvais contact des balais rendant souvent l'amorcement pénible. L'on peut aussi avoir recours à une petite batterie auxiliairc d'accumulateurs servant à exciter les inducteurs des moteurs-série pendant la période de freinage $\left(^{*}\right)$.
(A suipre)
J. Bethlinod.

\section{L'ÉLECTROCHIMIE A SAULT-SAINTE-MARIE}

Nous extrayons de la savante Revuc L'Elcctrochimie, que dirige avec tan d'autorite M. A. MinET, l'intéressante relation suivante de M. Joseph W. Richaros, président de la Société Electrochimique Américaine.

Une descriphon qui he se rapporterall qu'au lite ci-dessus sorant trop breve. Elle se redurall an procédé lhodin pour la pricparation de l'alcali caustrque el de la poudre a blanchr, car cest lo seul procedé installe it "The soo ". Avec lindulgence du fecteur, nous nous écarterons de celle queston étronle, et présenlerons sous ce trire une siluation génerale de Suall-Sante-Marice. essentiellement fivorable pour l'industrie électrochinuque.

Premerement, nous drions qu'on y rencontre une puissance hydradique fourne par la riviere samb-Nante gun prond sil source au lac supéreur qui. avec ses 30000 milles cimés, col ho plus grand réservorr d'eau du monde.

La ruviere est formée de rapides sur 111 espace d'environ III dami-mille et présente une chute de vingl pucds.

A sa source ello se presente dans de bornes condilions pour la construction d'un canal à cỏté des rapuldses, et conduire aitusi lieau ì une usine génératrice de pussance all-dessous dieux. liat quanlité d'eau varie aver la saison, les vents, mais elle esl all maximum de 3600000 pieds cubes par mimble representant unu puissance bydraulque de 130000 chevaux.

Colle-cr subura néanmoins une perte dans sa conversion en puissance mécarique, et une partic en est sacrifiéc pour amener l'eall à travers le canal de la prise a l'usme génératrice, finalcment celle derniere ne disposera que de dix-huit pieds.

Fn raison de cette observation il faul dire que le minimum de purssance utilisable a Sault-Sainte-Marie est d'environ 110000 chx.

On pourrait peul-être en prévoir davantage, mais cela ne valudrait gueve la peine d'mstallor une usine capable diune puissanm. dont elle naurat pas les éléments dutitisalion perdant une partio de l'année.

(*) Cet artifice a été essayé sur le réseau de tramwayslde Marseille. 
La présente utilisation de puissance comprend 20000 chevaux, fourms par une usine installéc sur le territore canadien, la proprété de "The Lake Superior Power $C^{\circ}$ " et de 40000 chevaux fourns par une usme construte sur le territore américan, la proprloté de "The Michigan Lake Superiol' Power Co ", de sorte que 60000 chevaux sur les 110,000 disponbles sont déji prets.

L'usine américaine vient a neine d'être achevée. Le canal a une longueur de deux milles et traverse la ville de Sault-Sante-Marie, Michigan.

Il a 200 pieds de large, 25 a 27 pieds de profondeur ; parcourt en parlie des terres siliceuses ronges, en partie d'argile rouge, et est capuble de dériver 20000 pieds cubes d'eau par seconde.

Il a été trés couteux a construre, le fond étant, par places, établi de facon à éviler le recurage el la construction de huit ponts ayant été reconnue indispensable aux endrolts où 11 crorse des rues.

L'usine génératrice est établie sur le bord de la rvière; elle a 1310 pieds de long, 87 piets de lange et 107 preds de haut; elle est batte avec une espece de prerres rouges des plus dures, avec des fondations sur plles.

Elle est diviséc en quatre-vingt paltres, dans chacuno desquelles sont installées quatre turbunes d'une puissance de 125 chevaux chacune, actionnunt une dynamo de 500 chevaux fixée sur l'étage au-dessus.

Le courant destmé aux industries voismes sera distribué à un faible voltage et avec un amperage approprlé; pour les usines ou les applications éloignées. il sera donné à un haut voltage.

Lo canal coute 3000000 dollar's : l'usine génoratrice, avec ses magasins et accessones, 1000000 dollars.

Si nous estumons ì $10 \%$ l'intéret que celle dépense rapporterat, le cout de l'entretien ef de la main-d'ueuvre ì $2,5 \%$, el l'amorlissement a $2,5 \%$, le prix de la puissance seralt d'environ $\mathbf{1 5}$ dollars par cheval électrique-aunèe.

Les exposés de la Comparme disent onliciellement que les industries qui feront des contrals à 20 dollars par cheval électriqueinnée, recevront des emplacements libres your leur usine, et seront exemptées des taxes municipales pendant cinq années; mais d'autres exposés non officiels sont courarts dans lesquels une parlie de la puissance est louée $\dot{a}$ un taux plus voism de 10 dollars que de 20.

L'usine canaluenne est siluée a cotś do l'écluse du Canada et avec un canal de 100 preds de large, elle distribue à présent 20000 chx.

Celtc pussance est presque entièrement employée, la plus grande partıe clant prisc par the Sante-Marle Pulp and Paper $\mathrm{C}^{\circ}$ qui possede lusine de pulpe de bols la plus importante du monde, foumssant 150 tonnes de pulpes fraiches, et 75 tommes blanchies l'anhydrude sulfureux.

Une intéressante combinasson est l'mstallation d'une usine pour griller les minerais de nickel de la Compagnie des mines do nockel a Sudbury.

Cette usme est placée le long des usines des pulpes de bois lanhydride sulfureux proluit par le grillage des minerais est dirigé directement à l'usine des pulpes et li ululisé pour le blanchiment et la gólatmisation des pulpes, colles-ci btant mises au contact de lhyposulfite de chaux.

Une fonderie de fer, un grand atelier mécanique emploient aussi une jussance considérable.

L'usine "énératrice et les autres constructions ont un bel et solide aspect, et il reste disponible beaucoup de terrains pour de nouvelles industries.

A environ un mille plus loin sur la rivière existe une nouvelle usine à acier, comprenant deux fours a vent pour produire du charbon de bois dur avec régénération d'acıde acétıque et d'esprit de bois: l'un des fours donne du coke pour fer, l'autre pour l'utihiser, el un Bessemer qui fournit 900 tonnes dacier par jour. C'est de ces usines que sont sortis les premers aciers laminés du Canada, depuis que le souvernement Canadien donne un boni de 3 dollars par tonne de fonte obtenue avec les minerais du Canada, et un boni additionnel de 5 dollars par tonne d'acier manufacturé au Canada; il n'y a aucun doute sur les résultats commerciaux de cette usine moderne.

Le procédé Rhodin pour la production de l'alcali caustique est le seul procédé électrochimique appliqué maintenant a Sault Sainte-Marle, et rous allons le décrire complètement.

Avant de faire cetle description hous dirons cependant qu'un contrat pour une puissance de 10000 chevaux est déja signé pour une manufacture de carbure de calcium, sur le territoire apéricain, pas the Union Carbide $\mathrm{C}^{\circ}$, et de 5000 chevaux pour l'extension du procédé Rhodın, tandis que dautres contrats sont en projet pour liblablissement d'usines blectrolytiques de raffinage du cuivre ef lu nokel de Sudbury.

Il est posstble que quelques-unes de ces propositions deviennent effectives.

Le carbure de calcium est d'un usage très répandu dans le Cànada occidental, ains que l'ont observé quelques auteurs dans un récent voyage à Victoria $B$. C

On estime que la consommation de ce produt augmentera con- siuérablement, car l'éclairage à l'acétylène convient bien aux conditions prévalantes dans ces vastes lerrituires.

Le cuvre du lac Supérieur raftiné peut ètre expédié très économiquement, de ce point, aux marchés de l'Est ; il en est de méme pour le nickel de Sudbury. Il est vrai de dire que les transports par bateaux sont interrompus pendant cinq mois de l'amıée, mars les chemins de fer établis pour Chicago ol autres points trans portent les marchandises a un prix assez bas pour contrebalanear pratiquement ce désavantage, et même pour faire le trafic nussi bon marché que sill étant operé toute l'année par le transport par cau.

Ce renseignement a été donné à l'auteur par M. F.-J. Clerrup directeur genéral de the Clerque syndicate et est néanmoins of clel.

The Canadian Electrochemical $C^{\circ}$ Ltd retire sa puissance motrice de the Lake Superior Power $\mathrm{C}^{\circ}$, sur le territoire Ontario, et est installée dans des bâtiments appartenant à cette Compagnie, dé. pendant de l'écluse conadienne.

Elle exploite le procédé Rhodin d'électrolyse du chlorure de sodium pour produire la soude caustique et la poudre de blan. chiment.

Lorsqu'elle fut visitée par l'auteur le 12 septembre 1902, ell n'étant pas en exploitation, parce que la guerre entre les Compagriles chımiques el électrochimiques d'Eurone avait tellement de. moralısé le marclié pour ce qui concerne ces produnts la poudre a blanchir étalt vendue par elles, au Canada, au prix très bas de 20 dollars la tonne) que les nouvelles usines ne pouvalent tra. vailler économiquement.

L'installation que nous allons décrire état considérée, par la Compagnie, comme plus ou moins expérimentale, et comme devant se développer davantage du coté américain.

Mais blen que la Compagnie américaine alt fait un contrat pom la location de la puissance, elle n'a pu construire une usine, en lauson de difficultés financières, n'ayant pu réaliser qu'un tron faible capital.

Il faut souhaiter qu'une réorganisation de cette Compagnie lui permette d'exploiter dans un avenir prochain.

Lusine canadienne comprend trois turbines de 350 chevay, actionnant chacune une dynamo de 200 kilowatts. Chaque machine fournil le courant à une série de quarante appareils électrolytiyues en séric, et la force électromotrice du courant pour chaque machine est de 220 volts, dont 20 volts pour vaincre la résistance des conducteurs et des connexions, et 200 volts pour le travall dans les appareils électrolytiques, soit 5 volts pour chacun de ces derniers.

Le princine des réactions dans les appareils est la décomposition du sel marin entre des électrodes en graphite et une couche de mercure comme cathode, répandue au fond d'une grande cuve en fer, les anodes étant comprises dans un vase en forme de cloche, qui condense et dégage au dehors le chlore produit, tandis que le mercure, chargé de sodium, s'échappe sous les borls de la cloche ol passe dans un espace exlérieur où il se trouve en contact aveo de l'eau; il y perd son sodium en produssunt la solution alcaline.

Un dispositif mécanique donne à la cloche un mouvement do l'otation, en meme temps qu'un flux et reflux continuel de solvtıon saline dans l'intérieur et une continuelle ou intermittente alimentation d'eau à l'extérieur de la cloche.

L'intérieur est isolé de l'extérieur par le fait que les bords dét la cloche plongent dans le mercure.

La cuve de fonte on de fer qui recoit la couche de mercure a 5 pleds de diamétre et 1 pled de hauteur; la couche de mercure 1 pouce d'épaisseur, le poids du mercure est de 1000 livres, re présentant une valeur de 600 dollars, ce qui constitue la plus grande dépense d'établissement d'un appareil.

Dans l'intéricur de la cuve est suspendue une cloche renversée en tcrre refractaile de 3 pieds de diamètre à travers le fond de laquelle passent les anodes qui sont en graphite, descendant jus. qu'a environ 1 pouce du mercure.

Les anodes sont fixées dans des ouvertures par un joint de ciment et sont en connection électrique avec des barres de cuiver. rui recoivent le courant par l'intermédairc d'un balai métalliqu (it cause de la rotation de la cuve).

Celle-ci est remple particllement d'une solution de sel marin, qui est renouvelée continuellement par un canal d'entrée et wh canal de sortie passant à travers le fond de la cuve.

La solution fratche est directement amenée dans le champ de dicomposition. entre les charbons et le mercure.

La cloche est supportée par une charpente qui s'appuie sur th bords supérieurs de la cuve et elle se prolonge par un cylindrh avec un rebord de diamètre un peu plus grand auquel fait suite tube de dégagement pour le chlore.

Pour assurer entre le cylindre et le tubc un joint étanche, of plonge les bords de ce dernier dans une rainure circulaire creuse dans le rebord du cylindre rempli d'eau, cette disposition per mettant la rotation de la cloche, le tube de dégagement restan fixe; cette ratation est obtenue au moyen d'un filet de vis et un 
d'ongrenage, et par elle, les anodes sont continuellement en roul dence de surfaces fraiches de mercure.

La solution de sel marin reçoit aussi un mouvement de rotaLa sui se communique ultérleurement au mercure, et ce dellion, gut en rajson des rainures radiales creusécs dans le fond de la nier, en rason est continuellement dirigé contre les paros de cette cure

cuve. lextérieur de la cloche se trouve une conche d'eau sur to mercure, qui agit sur l'amalgame et produl la soude caustique, mer dégagement dhydrogène.

clte solution esl maintenue jusqu'a ce qu'elle soit suffisamment concentrée; elle est alors ratirée par un siphon et remplacée par de leatu fratche.

les inventeurs ont prévu cque la cuve serant chaulfée jusqu'a une température voisine du point d'évaporation des solutions, cela friml avorr une solution de sel marin plus concentrée et aussi pour audmenter l'attaque de l'amalgame et diminuer la résistance de lappareil.

Cetla prévision était cependant une erreur, car au-dessus de Ti derrés 1 l se forme du chlorate en grande quantité, les anodes soit détruites et il y a une grande chance d'explosion à causc du mólange de chlore et d'oxygene qui se forme.

Le fait récl est que le passage de 990 ampères, par exemple, dans un tel appareil de 3 pieds de diamètrc, chaudfe le liquide en mison de sa propre résistance, plus qu'il ne convient, et que le probleme serait plutot de refroidir l'appareil que de lo chauffer.

La différence de potentiel théorique nécessaire à la décomposilion d'une solution saturée de sel marin, pour former du sodium et dégager du chlore est $\frac{97200}{23040}=4,22$ volts (97200 étant la chalemr de formation du chlorure de sodium en solution concentrée af 23040 la quantité de chaleur correspondant à 1 volt). Toutefis, l'absorption du sodium par le mercure et la formation d'amalginic dégaçe une quantité considérable d'énergie qui diminue le voltage théorique de $\frac{21600}{23040}=0,89$ volts (21 600 étant la chaleur de formation de l'amalgame). Le voltage nécessaire pour la dé composition du sel en solution concentrée est finalement de 3,33 volts. Le voltage pratique actuellement établi pour forcer un couranl de 900 ampères à traverser l'appareil est de 5 volts, dont 1.67 est nécessaire pour vaincre la résistance ohmique de l'appareil el représente l'énergie électrique convertic en chaleur.

C'est l'équivalent de $\mathbf{4 5}$ calories-livres par minule dans l'appareil. et c'est suffisant pour chauffer son contenu d'environ $20^{\circ} \mathrm{F}$. en une heure, en ne supposant aucune perte par radiation. Nous vovons, par conséquent, qu'il est tout à fait probable que l'appareil atteindra $20^{\circ} \mathrm{F}$. au-dessus de la température extérieure simplenent du fat de la chaleur dégagée par le passage du courant. Ilans un tel appareil il est probable qu'il $y$ aura quelque perte du lait de la recombinaison du sodium dans le compartiment anodique.

On n’a prévu aucun dispositif spécial pour le renouvellement de l'amialgame ni aucune methode pour le décharger au dehol's.

Ces deux opérations se produisent par elles-memes, pour ainsi dire, al la suite de l'action de charge.

i. Rhodin a établi que la perte résultant de la redissolution au contact du sel étail de $3 \%$.

On pouvait s'atlendre à ce qu'elle fủt plus grande. La déchargo est voltaique de sa nature; car la cuve en fer et l'amalgame forment ur couple voltalque fermé en court-circuit, qui, au conlact de l'eau, entraine la décharge du sodium et le dégagement do lihydrogène au contact du fer.

Cette action diffère de la méthode de décharge de lamalgame de Keller, en ce que cette dernière utilise une plaque cathodique disincte dans la solution caustique, séparée de l'amalgame et non en contact physique, cel ensemble constituant une pile galvanique dont l'amalgame est un pole, et la cathode l'autre pole, et de laquelle un courant galvanique peut être produit et utilisé, ainsi que le fait Keller d'après son brevet allemand, soit pour renforcer le eouranl principal de aécomposition, soit pour toute autre action independante.

Ivec l'action du couple voltaique de Rhodin, la force électromotrice engendrée n'est pas utilement applicable; l'énergie tout enthre de la décomposition de l'amalgame, représentant le cinpaème de l'energie électrique absorbée par l'ippareil, surchauffe

a soude caustique et augmente ainsi la tompérature de l'appareil.

L'arrangement adopté par Kellner et Castner, est tel que la

ferce électromotrice de decharge est renduc au circult. Mais une

partie de l'énergie récupérée par ce fait est convertie cn chaleur

dans la soude caustique pour vaincre la résislance ohmique entre

anode ct la cathode correspondant a cette solution; ajoutons

qu'avec une bonne disposition de cathode, une partie de la force

Linstalrice de décharge reste encore disponible.

Linstallation complète comprend 120 appareils, mais 80 seule-

liusint mis en action a la fois.

Lusine de la poudre à blanchir comprend 10 chambres de
55 pieds 6 pouces de long, 17 pieds de large et 6 pieds 6 pouces de haut, conshtuées de feuilles de plomb $\mathrm{n}^{\circ} 6$, avec un plancher un tule d'un demi-pouce. L'usine à chaux peut fournar lí tonnes de chaux éteinte par jour.

$\Lambda$ vec 80 apparels en achon, la production est de 1,4 tonnes de poudre a blanchir et 2 tomes de' soude caushque par jour.

Cecl représente onviron $80 \%$ de la production theorique rap. portée au courant dépensé.

Le sel employé contient $99.5 \%$ de chlorure de suclium, le reste ctant en grande partie consliluee par des sulfates de calemum et de sodium.

Ces sels, en raison de leur décomposition ef le chlore libre entrainent la dissolution de 1 à 1,20 pour cent de mercure dans la solution de sel marm, mais comme la solution cet sans cesse on travail sa concentration étant mainlcnue conslante par l'alimentator de sel nowvan, la perte résultant de ce fait est faible, satuf Iorsque la solution est changese, lon'squ'elle devient trop impure, et alors, chaque tros ou quatre huilaines environ, 20 livros de mercure (r0 francs) sont peldus. Dans la solution alcaline une livro de morcure (valeur 3 fr. 50 ) esl dissoule nour 27500 livres de produit causlique. Cette perte est donc négligeable an point de vue commercial.

Avec les 120 appareds on action, on arrwera a produire 4 lonnes de soude caustique et 9 tomnes de poudre a blanehir par jour; celie derniere quantité sufira ì la consommation du Canada: li premiele a une groude parlic de cotle nemo consommation, mats élant domé le pix de ces moduils sur lo marché au Cimada, l'usme ne saurat se maintenr avec profit, sans un larif de protection.

Nous apprenons, et nous sommes heureux d'en informor noslecteurs que l'exploitation des compteurs electriques stanicy " pour courants alternatifs, dont la description detallee a été donnéc dans le numbo io décembre 1903 de la Revue, vient d'utre confiée pourla krance à la liom. pagnje Westinghouse. C'est un nouveau gage de succes pour ces appareils déjà très répandus à l'ćtranger.

\section{La Houille blanche en Italie}

Dans le numéro de février 1904, La Houille Blanche a donné une "Statistique des concessions d'eau en Italie » qui se rapportait à l'ensemble des concessions accordées par l'Etat dans tout le pays. Nous nous proposons aujourd'hui de donner ici le résumé d'un rapport que le Ministère de l'Agriculture, de l'Industrie et du Commerce de Rome vient de faire paraître sur les richesses en énergie hydraulique de l'Italie centrale. On pourra ainsi apprécicr l'importance des disponibilités de houille blanche dont on pourrait tirer parti dans cette région.

Ce rapport évalue à $300000 \mathrm{HP}$ la puissance utilisable du Tibre; sur ce chiffre, on ne prévoit encore l'emploi que de 100 ooo HP. D'autre part, le Garigliano, le Volturno, le Sarno, le Tosciano, le Sele seraient capables de fournir 180 ooo HP sur lesquels on n'utilise actuellement que 35000 HP. De plus, on pourrait tirer des rivières Marna, Flora, Lombrone et Tronto 2 I 2 ooo HP, alors que l'on met à profit, actuellement, à peine la dixième partie de cette dernière puissance.

Le même rapport évalue à 767000 HP au total la puissance que l'on peut emprunter aux cours d'eau de l'Italie centrale. Comme il reste encore une disponibilité de 592000 $\mathrm{HP}$, on se rend facilement compte dés conséquences économiques que peut avoir, pour l'Italie, dont le sous-sol est très pauvre en houille noire, la mise en valeur de forces naturelles aussi abondantes.

Nous donnerons sous peu le résumé d'une conférence faite à la section milanaise de I'Association Electrotechnique Italienne sur des phénomènes d'instabilité observés dans des couplages d'alternateurs et de moteurs synchrones. 